Supporting Information

\title{
Cross-linked Nonwoven Fibers By Room Temperature Cure Blowing and in Situ
} Photopolymerization

Aditya Banerji, Kailong Jin, Kunwei Liu, Mahesh K. Mahanthappa, Christopher J. Ellison

Department of Chemical Engineering and Materials Science, University of Minnesota,

Minneapolis, MN 55455, United States

*To whom correspondence should be addressed: cellison@umn.edu

\section{Characterization methods and analysis:}

Fiber diameter measurement and analysis using visible light microscopy (VLM) and fieldemission scanning electron microcopy (FE-SEM) Cure blown fibers were imaged with a Nikon Optiphot visible light microscope equipped with a Canon SL1 digital camera and a Hitachi S-4700 FE-SEM. For VLM, the fiber samples were sandwiched between two clean glass slides and imaged in the transmission light illumination mode at a magnification of 10x. For FE-SEM, the fiber samples were coated with $\sim 7 \mathrm{~nm}$ layer of iridium using an ACE600 Coater. For each fiber mat, 710 SEM micrographs were taken with magnifications ranging from 50x-250x.

Statistical analyses were performed on 120-150 fiber diameter measurements from the VLM images using Image J $(\mathrm{NIH})$ software. It was verified that the results of the fiber diameter measurement and analyses varied less 5\% between the VLM and SEM images for a particular fiber mat sample. Due to the convenience of obtaining VLM images for the large number of fiber mats used in this study, all the statistical analyses were performed on the fiber diameter measurements from VLM. Origin Lab (a data analysis software package) software was used to fit a normal (or Gaussian) distribution function (Equation S1) to the fiber diameter data. The geometric average $\left(d_{\text {avg }}\right)$ and standard deviation $(\sigma)$ of the diameter distribution of the fiber mats obtained at different conditions were extracted from the corresponding normal fits.

$$
f(d)=\frac{1}{\sigma \sqrt{2 \pi}} \exp \left[-\frac{\left(d-d_{\text {avg }}\right)^{2}}{2 \sigma^{2}}\right]
$$




\section{Real time-Fourier transform infrared spectroscopy (RT-FTIR) and determination of gel}

time of the monomer mixture. The photopolymerization kinetics of the monomer mixture were studied by real time-Fourier transform infrared spectroscopy with a Thermo Fisher Scientific Nicolet 6700 FTIR spectrometer. A drop of the freshly prepared monomer mixture was spin coated on a $\mathrm{NaCl}$ window to obtain a thin film. The sample was prepared in a room with overhead light filtering ("an orange lighted room") to minimize any effect of the ambient light and loaded into the FTIR using a custom-made horizontal transmission accessory. The same light source as that used for fiber spinning experiments, connected to a liquid light guide with the collimator attached at the end, was used for RT-FTIR studies. Upon UV illumination, FTIR spectra were collected under ambient conditions with a time resolution of $0.04 \mathrm{~s}$ and a spectral resolution of $14.73 \mathrm{~cm}^{-1}$.

However, if the same high intensity light as that used in fiber spinning experiments was used for RT-FTIR studies, the gel point of the monomer mixture would be reached too quickly. Accurate measurement of the gel time was not possible for these conditions due to the spectrometer's time resolution limit. Therefore, RT-FTIR studies were performed at lower light intensities ranging from 38 to $215 \mathrm{~mW} / \mathrm{cm}^{2}$ to obtain a well-resolved evolution of the photopolymerization reaction. The fractional conversion of acrylate double bonds during UV exposure was monitored based on the reduction in the area of the characteristic acrylate $\mathrm{C}=\mathrm{C}$ peak at $1630 \mathrm{~cm}^{-1} \cdot{ }^{1-2}$ The acrylate double bond peak area normalized with the non-reacting carbonyl peak $\left(1720 \mathrm{~cm}^{-1}\right)$ area was used to obtain the conversion-time data at different light intensities (Figure S1 (a)). Previous studies by Cramer et al. showed a zero-order rate dependence of the initial rate of polymerization on the acrylate double bond concentration. ${ }^{3}$ Thus, at lower conversion, the conversion-time data can be fit linearly with the resulting equation being used to calculate the gel time. Figure S1 (b) shows the linear fits of the conversion-time data up to $25 \%$ conversion for a DPPA-PETT mixture with $5 \mathrm{wt} \%$ photoinitiator (the same amount as that in the mixture used for fiber spinning experiments) at light intensities of $153 \mathrm{~mW} / \mathrm{cm}^{2}$ and $261 \mathrm{~mW} / \mathrm{cm}^{2}$. From the linear fits, the gel times were calculated to be $9.8 \mathrm{~ms}$ and $5.5 \mathrm{~ms}$, respectively. 
(a)

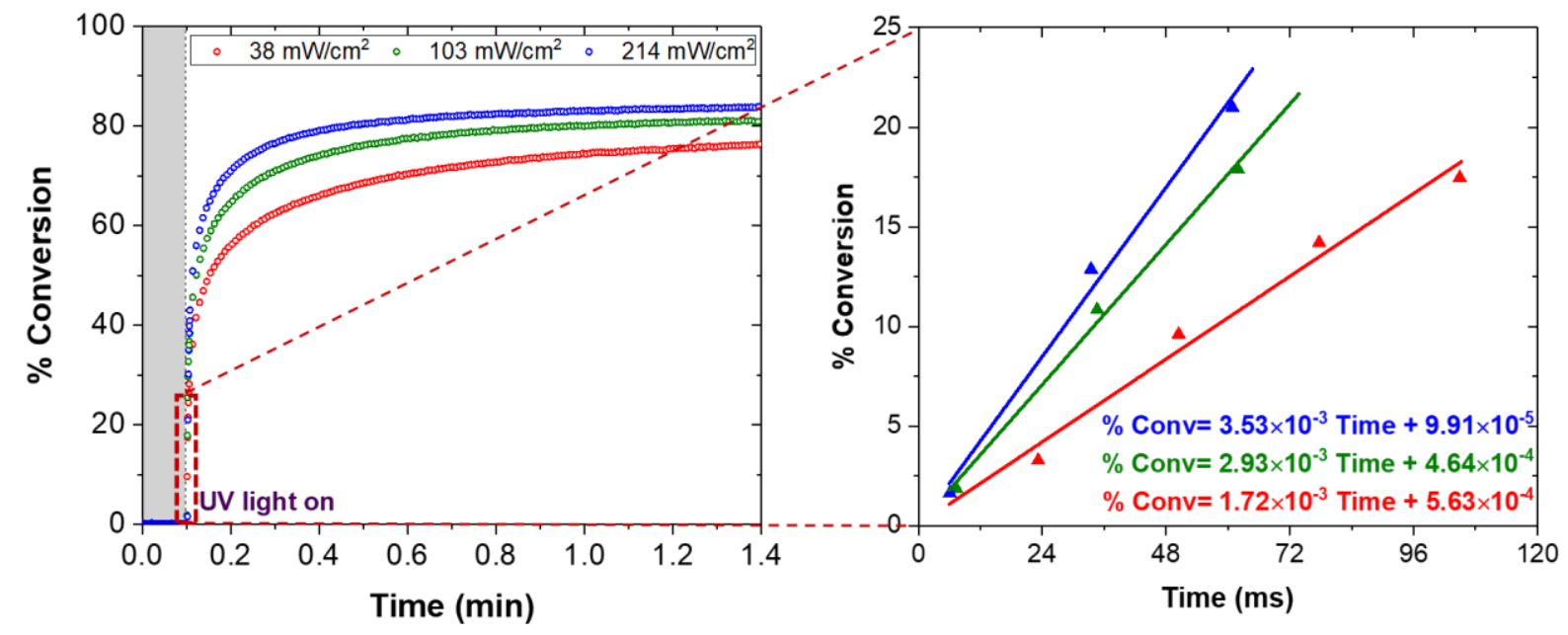

Figure S1. (a) Conversion-time data at different UV intensities obtained by monitoring the reduction in the acrylate double bond peak $\left(1630 \mathrm{~cm}^{-1}\right)$ area normalized by the non-reacting carbonyl peak $\left(1720 \mathrm{~cm}^{-1}\right)$ area. (b) Linear fits of the conversion-time data at low $\%$ conversion (magnified section in Figure S1 (a)) and the corresponding linear fit equations at the respective UV intensities.

The gel time at the light intensity used for fiber spinning experiments was extrapolated using the data obtained at the lower UV intensities. The dependence of the photopolymerization kinetics on the light intensity is required for proper extrapolation. For a classical free radical polymerization, the rate of polymerization $\left(\mathrm{R}_{\mathrm{p}}\right)$ is proportional to the $1 / 2$ power of the rate of initiation $\left(R_{i}\right)$, i.e., $R_{p} \propto\left(R_{i}\right)^{1 / 2} .^{4-5}$ For a photoinitiated polymerization, as is the case in this study, the initiation constant is in turn proportional to the light intensity $\left(R_{i} \propto I\right){ }^{5}$ Therefore, at the gel point (i.e., at a specific change in the monomer concentration), the gel time would be proportional to $\mathrm{I}^{-1 / 2}$. Figure $\mathrm{S} 2$ shows the variation of the gel times calculated at low light intensities as a function of $\mathrm{I}^{-1 / 2}$. The linear fit equation obtained from the data can then be used to estimate the gel time at the fiber process light intensity. 


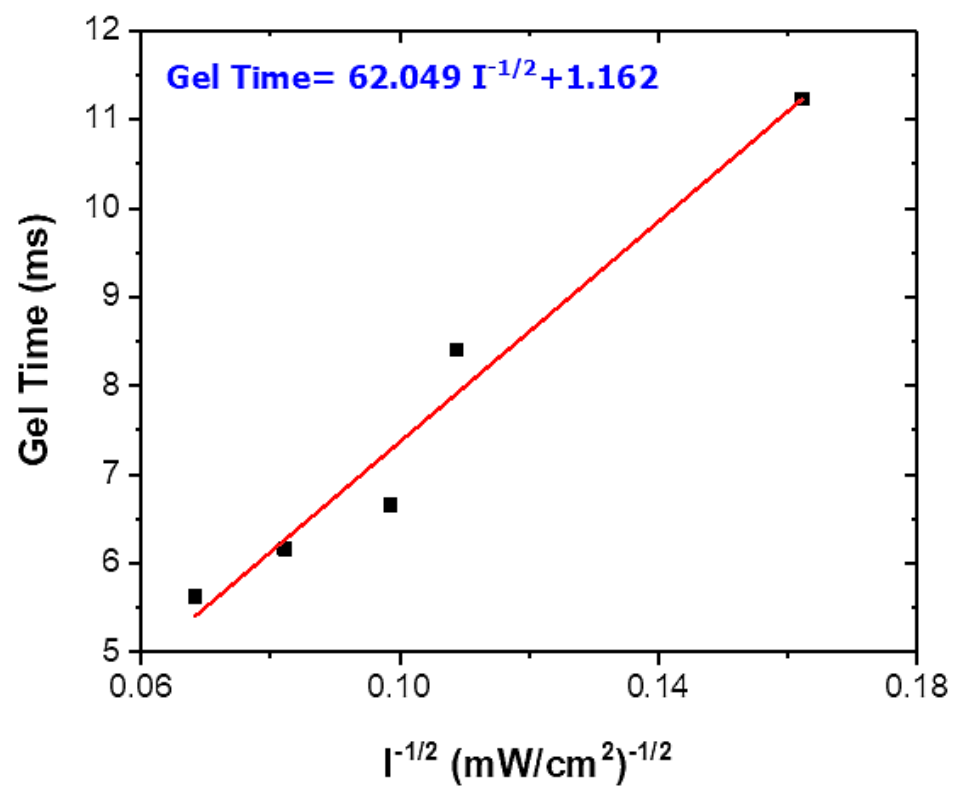

Figure S2. Gel times of the monomer mixture as a function of the UV intensities (I) obtained from polymerization kinetics studies using RT-FTIR. The solid line represents the best fit line through all data points.

\section{Characterization of monomer mixture absorption characteristics by UV-vis Spectroscopy.} The UV-vis spectroscopy absorption curves in Figure S3 were obtained using a Thermo Fisher Scientific Evolution 220 UV-visible spectrophotometer. One drop of the freshly prepared monomer mixture was spin coated on a $\mathrm{NaCl}$ window to obtain a $\sim 45 \mu \mathrm{m}$ thick film. Film absorption curves were measured both before and after UV exposure. Absorbance values were measured at $\lambda=365 \mathrm{~nm}$, which corresponds to the peak absorption wavelength of the Omnirad 2100 photoinitiator. Figure S3 shows that the unreacted monomer mixture exhibits an absorbance $A=0.13$, or $\%$ transmittance $(\% T)=74 \%$. Furthermore, after $\sim 20 \mathrm{sec}$ of UV exposure, the absorbance reduced to $A=0.07(\% T=85 \%)$. This result indicates that with the progression of photopolymerization, the depletion of the photoinitiator allows a greater extent of light penetration. Penetration depth is defined as the depth at which the intensity of the radiation inside the material falls to about $37 \%(1 / e)$ of its original value at the surface. Since the measured $\% T$ values are greater than the critical value, UV transmission issues are not expected to arise nor affect the extent 
of curing throughout the fiber cross-section for the range of attenuated liquid fiber diameters involved in cure blowing $(\sim 5-35 \mu \mathrm{m})$.

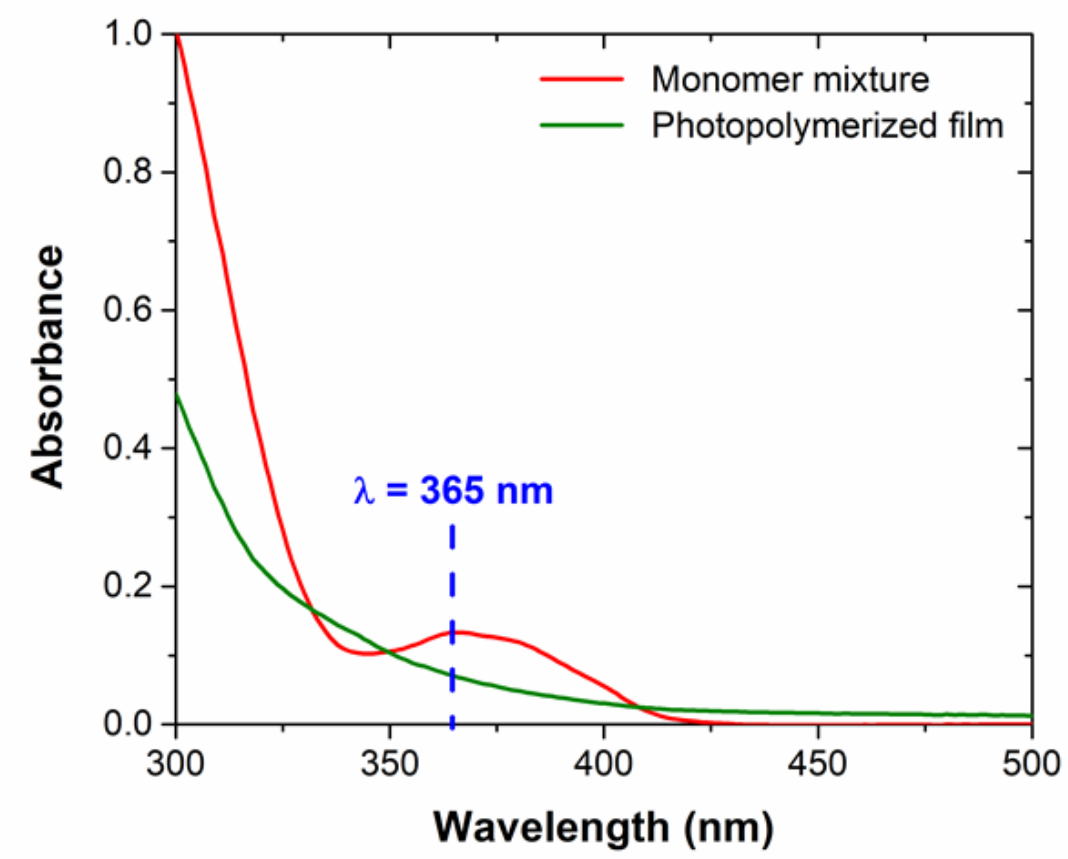

Figure S3. UV-visible absorption spectra obtained from a $\sim 45 \mu \mathrm{m}$ thick film of the monomer mixture before and after photopolymerization.

Estimation of UV exposure times. The fiber velocity profile during cure blowing was approximated by the power law model for air velocity profile in melt blowing developed by Moore et al. (Equation S2) using a similar die geometry as used in this study.

$$
\frac{v(z)}{v_{0}}=1.864\left(\frac{z}{h}\right)^{-0.6321}
$$

where $v(z)$ is the mean air velocity along the fiber spinning axis, $v_{o}$ is the mean air velocity at the die-face, $z$ is the distance from the die-face and $h$ is the sum of the orifice diameter and total air knife gap. The UV exposure time was estimated by integrating the approximated fiber velocity profile using proper limits. Since, both the fiber and air velocity profile decays in the UV illuminated region following a power law decay, the approximation should serve as a satisfactory estimation of the UV exposure time. 


\section{Simplification and analysis of the analytical expression for $\omega_{\max }$ from Equation 4.}

From Equation 4,

$$
\omega_{\max }=\frac{1}{2 \sqrt{2 C a}(1+3 S \sqrt{C a / 2})}
$$

where the retardation number, $\mathrm{S}$ and the capillary number, $\mathrm{Ca}$ are given by,

$$
S=\frac{1}{1+G \lambda_{E} / \mu_{S}} \quad C a=\frac{\rho v^{2}}{\sigma r_{0}^{2}}
$$

However, the characteristic viscosity, $v$ appearing in $C a$ is defined in terms of $S$ as $v=\mu_{s} / S \rho$. Hence, the expression for $\mathrm{Ca}$ simplifies to,

$$
C a=\frac{\mu_{S}^{2}}{\rho \sigma r_{0}^{2}} \frac{1}{S^{2}}=\frac{\mu_{S}^{2}}{\rho \sigma r_{0}^{2}}\left(1+G \lambda_{E} / \mu_{S}\right)^{2}
$$

Using the expressions for $S$ and $C a, \omega_{\max }$ is given by,

$$
\omega_{\max }=\frac{1}{2 \sqrt{2 \frac{\mu_{S}^{2}}{\rho \sigma r_{0}^{2}}\left(1+G \lambda_{E} / \mu_{S}\right)^{2}}\left(1+3 \frac{1}{\left(1+G \lambda_{E} / \mu_{S}\right)} \sqrt{\frac{\mu_{S}^{2}}{2 \rho \sigma r_{0}^{2}}\left(1+G \lambda_{E} / \mu_{S}\right)^{2}}\right)}
$$

which on further simplification is given by,

$$
\omega_{\max }=\frac{1}{\frac{2 \mu_{S}}{r_{0}} \sqrt{\frac{2}{\rho \sigma}}\left(1+3 \sqrt{\frac{1}{2 \rho \sigma}} \frac{\mu_{S}}{r_{0}}\right)\left(1+G \lambda_{E} / \mu_{S}\right)} \propto \frac{1}{\left(1+G \lambda_{E} / \mu_{S}\right)}
$$

From the simplified expression, $\omega_{\max }$ is inversely proportional to a linear function of $\lambda_{\mathrm{E}}$. 


\section{Supporting Results:}

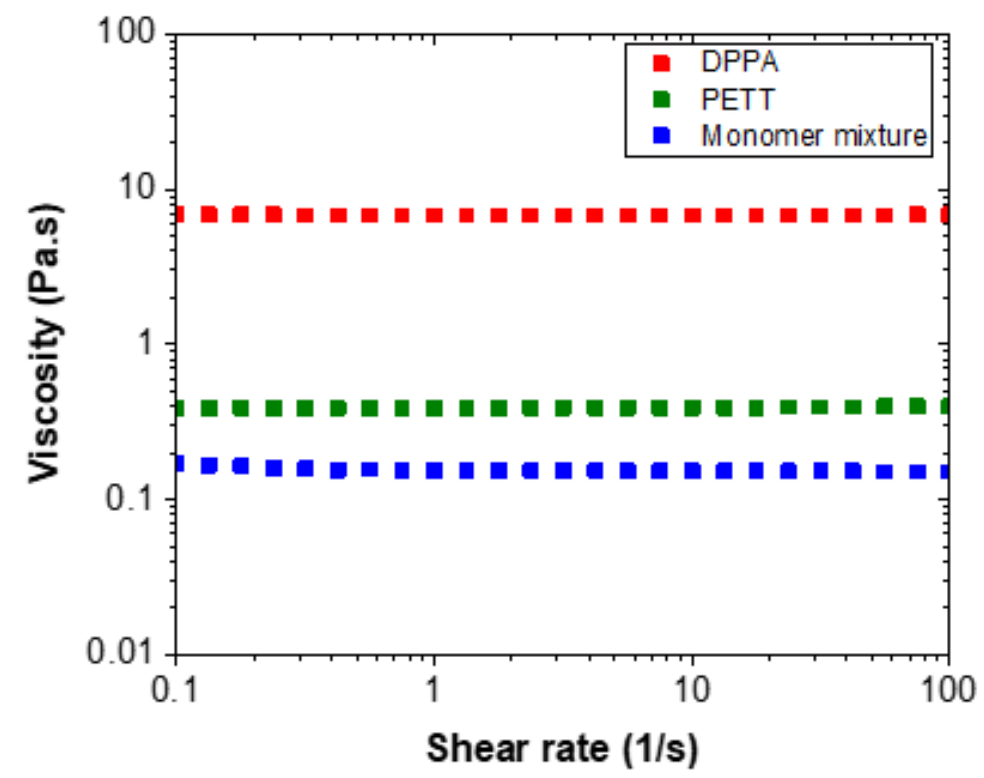

Figure S4. Viscosities of the DPPA, PETT and a monomer mixture with composition of 64 wt \% DPPA, $19 \mathrm{wt} \%$ PETT, $5 \mathrm{wt} \%$ Omnirad 2100 and $12 \mathrm{wt} \%$ ethyl acetate. 
(a)

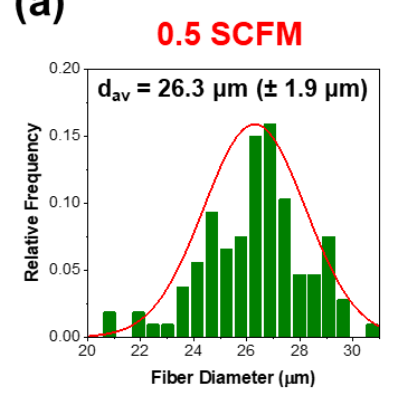

(b)

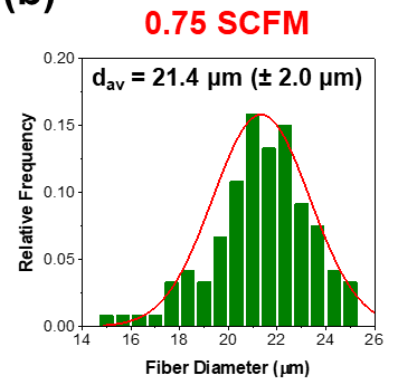

(c)

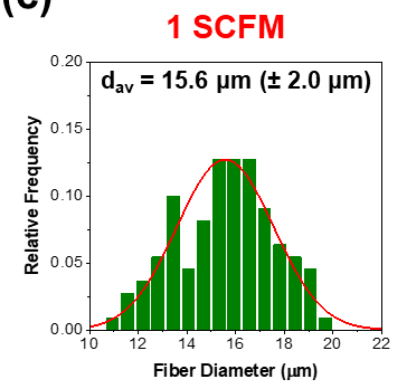

(d)

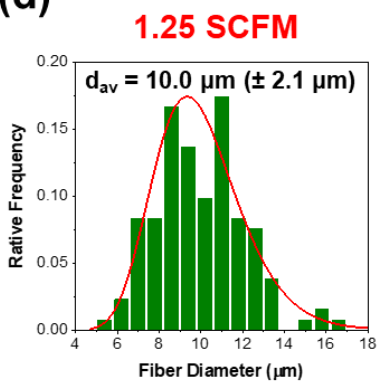

Air flow rate held constant

(e)

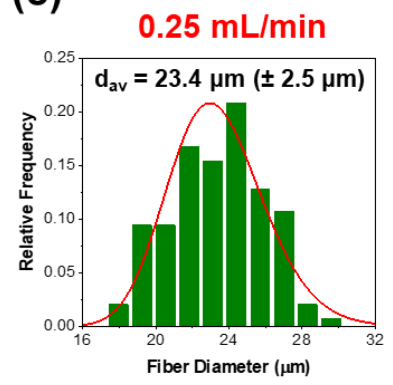

(f)

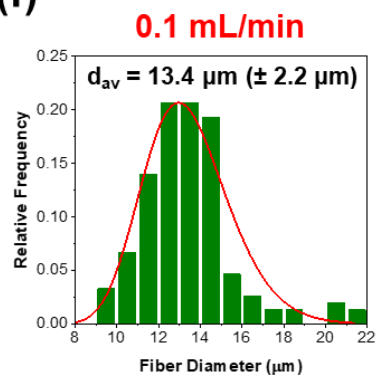

(g)

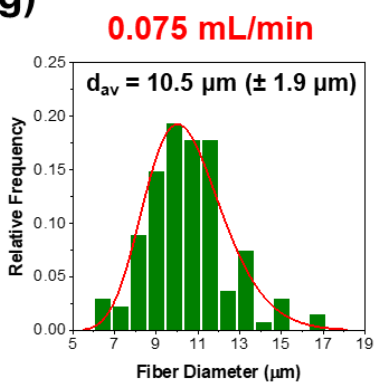

(h)

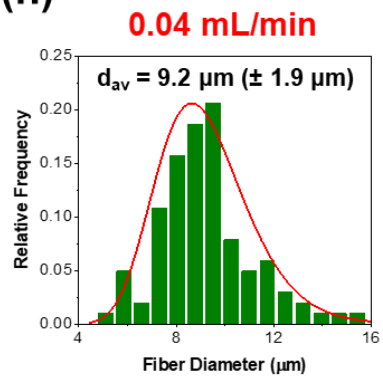

Figure S5. Statistical analysis of the fiber diameter obtained by variations of air flow rate and monomer delivery rate: (a)-(d) Analysis of fiber diameters obtained at a constant monomer delivery rate of $0.25 \mathrm{~mL} / \mathrm{min}$ with air flow rates of (a) 0.5 , (b) 0.75 , (c) 1 and (d) 1.25 SCFM. (e)-(h) Analysis of fiber diameters obtained at a constant air flow rate of 0.5 SCFM with monomer delivery rates of (a) 0.25 , (b) 0.1 , (c) 0.075 and (d) $0.04 \mathrm{ml} / \mathrm{min}$. The average and standard deviation of the fiber diameters obtained using a normal distribution in all the cases. 


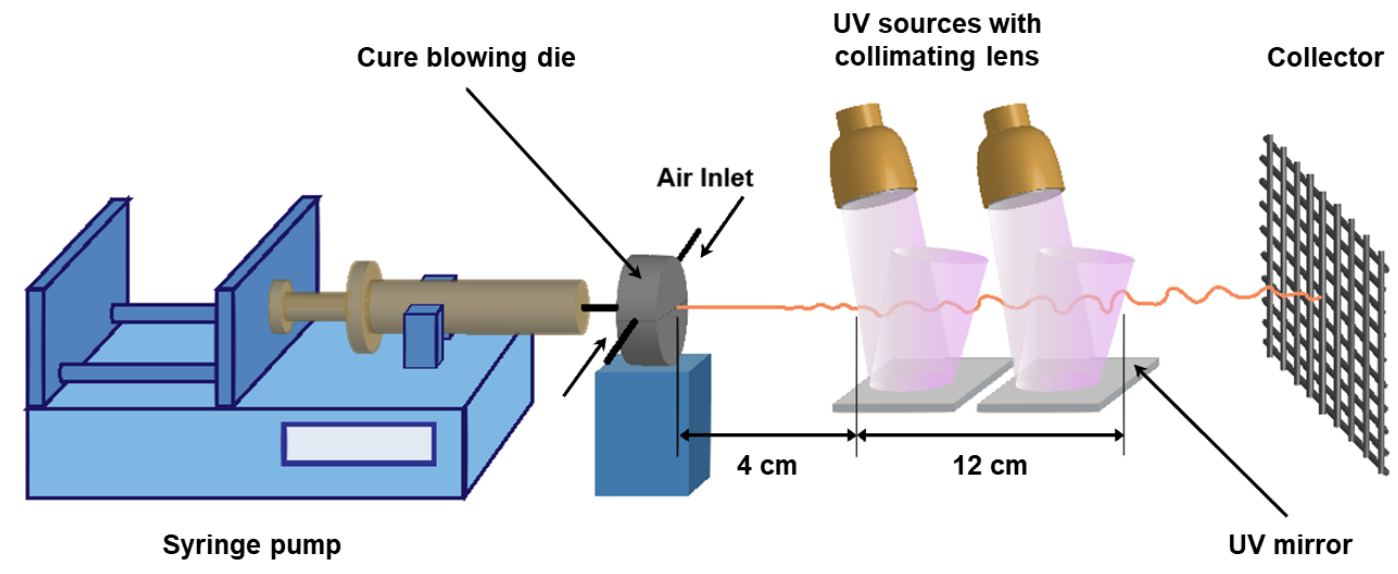

Figure S6. Schematic representation of the cure blowing apparatus with the modified light arrangement (2 UV sources and mirrors)
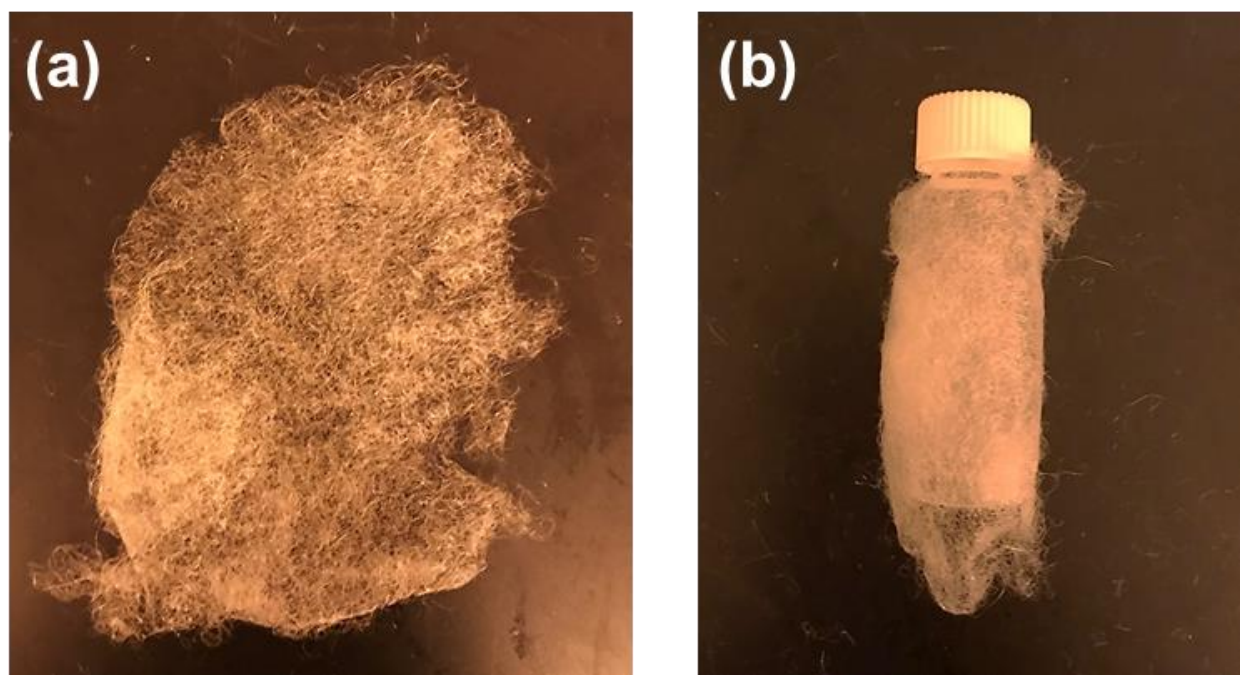

Figure S7. Representative photograph of (a) an as-obtained fiber mat and (b) the same fiber mat wrapped around a glass scintillation vial 


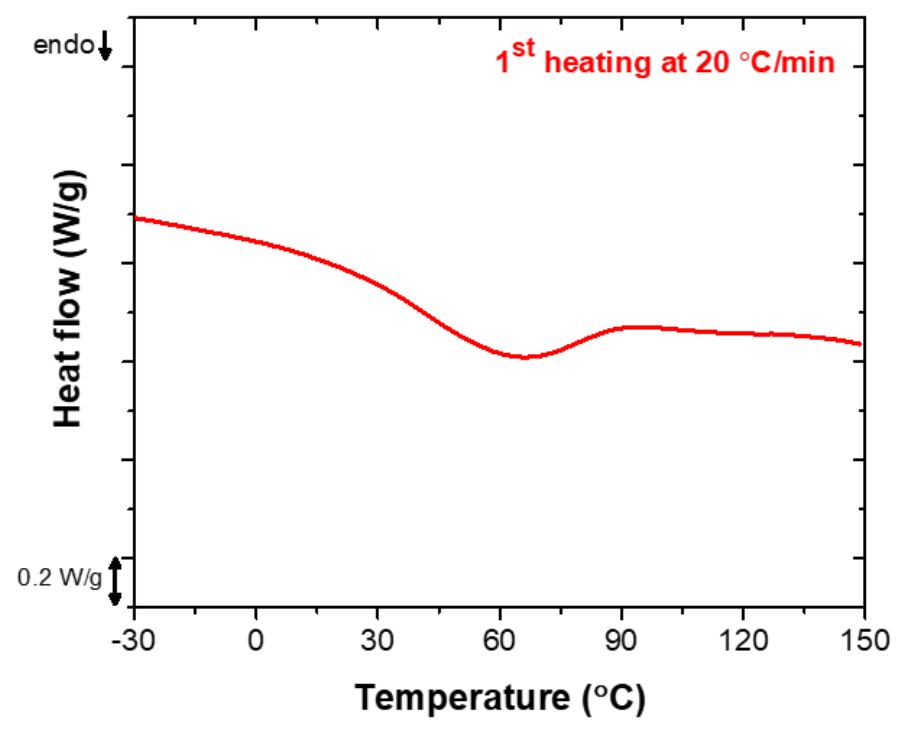

Figure S8. DSC thermogram of as-obtained cure blown fibers

Table S1: Summary of the estimated UV exposure times for the initial and modified light arrangements

\begin{tabular}{ccc}
\hline & \multicolumn{2}{c}{ UV exposure time (ms) } \\
\cline { 2 - 3 } Air flow rate (SCFM) & Initial light arrangement & Modified light arrangement \\
\cline { 2 - 3 } 0.5 & 3.7 & 13.0 \\
0.75 & 2.4 & 8.6 \\
1 & 1.7 & 6.2 \\
1.25 & 1.3 & 4.8 \\
\hline
\end{tabular}




\section{References}

1. Shanmuganathan, K.; Sankhagowit, R. K.; Iyer, P.; Ellison, C. J., Thiol-Ene Chemistry: A Greener Approach to Making Chemically and Thermally Stable Fibers. Chem. Mater. 2011, 23, 4726-4732.

2. Reddy, S. K.; Cramer, N. B.; Bowman, C. N., Thiol-Vinyl Mechanisms. 2. Kinetic Modeling of Ternary Thiol-Vinyl Photopolymerizations. Macromolecules 2006, 39, 3681-3687.

3. Cramer, N. B.; Davies, T.; O'Brien, A. K.; Bowman, C. N., Mechanism and Modeling of a Thiol-Ene Photopolymerization. Macromolecules 2003, 36, 4631-4636.

4. Hiemenz, P. C.; Lodge, T. P., Polymer Chemistry; CRC Press: 2007.

5. Anseth, K. S.; Bowman, C. N.; Peppas, N. A., Polymerization kinetics and volume relaxation behavior of photopolymerized multifunctional monomers producing highly crosslinked networks. J. Polym. Sci A Polym. Chem. 1994, 32, 139-147. 